\title{
Surpass Flow Diverter for Treatment of Posterior Circulation Aneurysms
}

(D).A. Taschner, (D). Vedantham, (D). de Vries, (D). Biondi, (D). Boogaarts, (D). Sakai, (DP. Lylyk, (D). Szikora, (DS. Meckel, (D). Urbach, DP. Kan, (DR. Siekmann, (D). Bernardy, DM.J. Gounis, and (D) A.K. Wakhloo

\begin{abstract}
BACKGROUND AND PURPOSE: Flow diverters for the treatment of posterior circulation aneurysms remain controversial. We aimed to identify factors contributing to outcome measures in patients treated with the Surpass flow diverter for aneurysms in this location.
\end{abstract}

MATERIALS AND METHODS: We conducted an observational study of 53 patients who underwent flow-diverter treatment for posterior circulation aneurysms at 15 centers. Key outcome measures were mortality, complete aneurysm occlusion, and modified Rankin Scale score at follow-up.

RESULTS: At follow-up (median, 11.3 months; interquartile range, 5.9-12.7 months), 9 patients had died, resulting in an all-cause mortality rate of $17.3 \%(95 \% \mathrm{Cl}, 7 \%-27.6 \%) ; 7$ deaths (14\%) were directly related to the procedure and none occurred in patients with a baseline mRS score of zero. After adjusting for covariates, a baseline mRS of 3-5 was more significantly $(P=.003)$ associated with a higher hazard ratio for death than a baseline mRS of 0-2 (hazard ratio, 17.11; 95\% Cl, 2.69-109.02). After adjusting for follow-up duration, a 1-point increase in the baseline mRS was significantly $(P<.001)$ associated with higher values of mRS at follow-up (odds ratio, 2.93; 95\% $\mathrm{Cl}, 1.79-4.79$ ). Follow-up angiography in 44 patients (median, 11.3 months; interquartile range, 5.9-12.7 months) showed complete aneurysm occlusion in 29 (66\%; 95\% Cl, 50.1\%-79.5\%).

CONCLUSIONS: Clinical results of flow-diverter treatment of posterior circulation aneurysms depend very much on patient selection. In this study, poorer outcomes were related to the treatment of aneurysms in patients with higher baseline mRS scores. Angiographic results showed a high occlusion rate for this subset of complex aneurysms.

ABBREVATIONS: $\mathrm{BT}=$ basilar trunk; $\mathrm{FD}=$ flow diverter; $\mathrm{HR}=$ hazard ratio; KWANOVA = Kruskal-Wallis analysis of variance; $\mathrm{PCA}=$ posterior cerebral artery; $\mathrm{Q} 1-\mathrm{Q} 3=$ quartiles $1-3 ; \mathrm{VB}=$ vertebrobasilar

Elow diverters (FDs) have proved to be reliable tools for the treatment of complex aneurysms, ${ }^{1}$ but their use for aneurysms

\section{Received August 3, 2016; accepted after revision October 11}

From the Department of Neuroradiology (C.A.T., S.M., H.U., J.B.), Medical CentreUniversity of Freiburg, Freiburg, Germany; Department of Radiology (S.V.), New England Center for Stroke Research (M.J.G.), and Division of Neuroimaging and Intervention (A.K.W.), Departments of Radiology, Neurology, and Neurosurgery, University of Massachusetts Medical School, Worcester, Massachusetts; Department of Neurosurgery (J.d.V., J.B.), Radboud University Nijmegen Medical Center, Nijmegen, the Netherlands; Department of Neuroradiology and Endovascular Therapy (A.B.), University of Besançon, Besançon, France; Department of Neurosurgery (N.S.), Kobe City Medical Center General Hospital, Kobe, Japan; Department of Neurosurgery (P.L.), Equipo de Neurocirugía Endovascular Radiología Intervencionista, Buenos Aires, Argentina; National Institute of Neurosciences (I.S.), Budapest, Hungary; Department of Neurosurgery (P.K.), Baylor College of Medicine, Houston, Texas; and Department of Neuroradiology (R.S.), Klinikum Kassel, Kassel, Germany.

We obtained a travel grant for monitoring visits from Stryker Neurovascular $(\$ 1100)$. Trial Registration: http://www.germanctr.de. Unique identifier: DRKS00006881. Preliminary data from this study were presented at: Anatomy, Biology, Clinical Correlations - Working Group in Interventional Neuroradiology (ABC-WIN) Seminar, January 18-23, 2015; Val d'Isère, France; the American Society of Neuroradiology Annual Meeting and the Foundtion of the ASNR Symposium, April 25-30, 2015; in the posterior circulation remains controversial. ${ }^{2-4}$ The large number of perforating and branching arteries of the posterior circulation potentially increases the risk in flow diversion, exposing patients to thromboembolic complications and brain stem stroke. FD treatment of giant, fusiform aneurysms of the vertebrobasilar (VB) junction or basilar trunk (BT) has been associated with high morbidity and mortality of up to $71 \% .^{2-4}$

A recent meta-analysis identified 14 studies, which reported on 225 posterior circulation aneurysms treated with FDs in 220 patients. ${ }^{5}$ The procedure-related good outcome rate was $79 \%$ (95\% confidence interval, $72 \%-84 \%$ ), and the procedure-related mortality rate was $15 \%(95 \% \mathrm{CI}, 10 \%-21 \%)$. Most studies in-

Chicago, Illinois; and the Annual Meeting of the German Society of Neuroradiology, October 15-17, 2015; Cologne, Germany.

Please address correspondence to Christian A. Taschner, MD, Department of Neuroradiology; Medical Center-University of Freiburg, Breisacherstr 64, D-79106 Freiburg, Germany; e-mail: christian.taschner@uniklinik-freiburg.de

-- Indicates open access to non-subscribers at www.ajnr.org

三 Indicates article with supplemental on-line tables.

http://dx.doi.org/10.3174/ajnr.A5029 
cluded only a small number of patients (median, 9.5; range, 5-55), and most of these patients (155/220) were treated with the Pipeline Embolization Device (Covidien, Irvine, California).

The Surpass FD (Stryker Neurovascular, Fremont, California) has various features that seem to predetermine its use in the posterior circulation: 1) The maximum length of $50 \mathrm{~mm}$ allows aneurysm treatment with a single device, thus avoiding telescoping; 2) due to the rhomboid shape of the FD cells, the flow-diverting effect remains constant in tapered vessels. The rhomboid shape allows a consistent cell shape across a wide range of vessel diameters. Specifically, this feature enables consistent porosity and pore density along the length of the implant in a tapering vessel. These are known variables that contribute to flow diversion and thrombosis of the aneurysm.

On the other hand, the Surpass FD has a particularly high mesh density compared with the Pipeline Embolization Device, potentially increasing the risk of perforator strokes. ${ }^{6}$ The purpose of this study was to identify factors contributing to outcome measures in patients treated with the Surpass FD for aneurysms located in the posterior circulation.

\section{MATERIALS AND METHODS \\ Study Design and Participants}

This multicenter, retrospective, observational study was approved by the appropriate ethics committee of the lead institution (Faculty of Medicine, University of Freiburg) and was registered in the German Clinical Trials Register (DRKS-ID: DRKS00006881). In Europe, patients were treated with the Surpass FD after it received approval for distribution by the Notified Body (CE Mark); outside Europe, patients were treated under a compassionate use protocol. Patients were entered prospectively into a data base if they had a posterior circulation aneurysm for which treatment was attempted with the Surpass FD. The design and technical specifications of the Surpass FD have been detailed elsewhere. ${ }^{7}$ Interventional procedures with the Surpass FD were performed in accordance with local institutional guidelines at each participating center. Before the procedure, each patient was given dual antiplatelet therapy. When possible, this consisted of clopidogrel, $75 \mathrm{mg}$, and aspirin, $325 \mathrm{mg}$, for 5 days. In the acute setting, patients were routinely given a loading dose of clopidogrel, $600 \mathrm{mg}$, and aspirin, $325 \mathrm{mg}$, the night before the operation.

Patient demographics and aneurysm characteristics were obtained from medical charts. Technical success, complications, clinical outcome, and imaging follow-up were determined.

\section{Data Collection}

Collected data included age, sex, modified Rankin Scale score at baseline, aneurysm location, aneurysm size (height, width, and depth) and morphology, neck diameter (in fusiform aneurysms, the length of the affected vessel segment was considered the aneurysm neck), previous treatment attempts, rupture status, number of FDs, additional coiling, procedural complications, new neurologic deficits 24 hours posttreatment, mRS score at discharge, mRS score at last clinical follow-up, duration of clinical follow-up (months), angiographic evaluation of aneurysm occlusion by using Kamran or Raymond scores, time point of angiographic follow-up, and date of death (if applicable). Aneurysm location was defined as a categoric variable representing the VB junction, BT, V4, and posterior cerebral artery (PCA).

Data were missing for age and sex in 1 patient; aneurysm size, in another patient; and neck diameter, in 4 patients. Two patients died before discharge, and 2 died after discharge (mRS scores at discharge: 1 and 5) and before the first follow-up. Of the remaining 48 patients, angiographic follow-up was available for 44 .

\section{Outcomes}

Clinical outcomes were graded according to the mRS at baseline (presentation), discharge, and follow-up. ${ }^{8}$ The primary safety and effectiveness measures were mortality and angiographic evidence of complete occlusion at the median follow-up of approximately 12 months, respectively. The secondary outcome measures were procedural complications, new neurologic deficits at 24 hours following the procedure, mRS score at hospital discharge, and morbidity defined as an mRS score of 3-5 at clinical follow-up.

\section{Statistical Methods}

Outcome Measures. The analyzed outcome measures listed in temporal order of assessment were procedural complications (binary), new neurologic deficit at 24 hours posttreatment (binary), $\mathrm{mRS}$ at discharge (ordinal), complete angiographic occlusion at last angiographic follow-up (binary), mRS at last clinical follow-up (ordinal), mortality (binary), and morbidity (binary) at last follow-up.

Data Preparation. Complete angiographic occlusion (binary) was defined as a Kamran score of 4 or a Raymond score of 1 . If mortality occurred and the date of death was known, it was specified as the last follow-up date along with $\mathrm{mRS}=6$. For the 2 patients who died after hospital discharge but before follow-up, the date of death could not be determined; hence, the date of last clinical contact was pragmatically assigned as date of death along with $\mathrm{mRS}=6$.

All analyses were performed by using statistical software (SAS 9.3; SAS Institute, Cary, North Carolina). For all final analyses, effects associated with $P<.05$ were considered statistically significant. Correlations (Spearman $\rho$ ) between all variables were assessed. When appropriate, the Sidak multiple comparisons adjustment was performed. Associations between procedural factors (number of FDs, additional coiling) and patient characteristics at baseline were assessed by using appropriate nonparametric tests. Binary outcome measures at fixed time points (procedural complications, new deficits at 24 hours posttreatment) were analyzed by using logistic regression models using the Firth bias correction. Variables that were significantly correlated with each binary outcome or variables that had $P<.2$ in univariate logistic regression were included in the corresponding multivariable logistic regression model that used stepwise selection. In multivariable models, interaction among predictors was evaluated and is reported only if significant. Hosmer-Lemeshow goodness-of-fit tests were performed.

Ordinal outcomes (mRS at discharge, mRS at last clinical follow-up) were initially modeled by using ordered logistic regression, and if the proportional odds assumption could not be satisfied, generalized linear models were fit by specifying a multinomial distribution and cumulative logit-link function. Variables that were statistically correlated with the outcome measure were 
included in the initial model, which was refined by sequentially excluding the variable with the highest $P$ value, provided that the $P$ value was $>.05$. The goodness-of-fit of the model was assessed by using the Pearson $\chi^{2}$ test.

Kaplan-Meier survival analysis and the Cox proportional hazards model were used to analyze the binary outcomes (complete occlusion, mortality, and morbidity) assessed at varying time points. For continuous covariates, survival analysis was performed by categorizing at the quartiles of each variable. All covariates that exhibited $P<.1$ in Kaplan-Meier analysis were included in the Cox proportional hazards model that used stepwise selection. For Cox proportional hazards models, cumulative Martingale residuals to check the functional form for the continuous variables and a standardized score process to check the proportional hazards assumptions were performed. Kolmogorov-type supremum tests were computed with 500 simulated patterns.

\section{RESULTS}

From July 2010 through March 2015, data from 53 consecutive patients with 53 acutely ruptured or unruptured aneurysms in the posterior circulation treated with Surpass FDs at 15 centers in 8 countries were entered into the data base. In 1 patient with a saccular vertebral artery aneurysm, tortuosity of major vessels prevented FD placement. Treatment with a different FD failed, and the aneurysm was treated with stent-assisted coiling. This patient was excluded from this cohort analysis, resulting in a final dataset of 52 patients.

\section{Baseline Characteristics}

Patient demographics, clinical presentations, and aneurysm characteristics are summarized in Table 1. Of 52 patients, 20 (38\%) were mRS 0,18 (35\%) were mRS 1 or 2 , and $14(27 \%)$ were mRS 3-5 at presentation. Of 52 aneurysms treated with the Surpass FD, $40(77 \%)$ were fusiform or dissecting. Seventeen aneurysms (33\%) were $>20 \mathrm{~mm}$. Seven patients presented with acutely ruptured aneurysms.

Figures 1 and 2 show case examples, and Fig 3 summarizes the results of the statistical analysis. The detailed results of the statistical analysis are available in On-line Tables 1-4.

\section{Procedures}

Technical success was achieved in 52/53 patients (98\%); coverage of the target aneurysm consisted of $1(n=35 ; 67 \%), 2(n=12$; $23 \%)$, or $3(n=5 ; 10 \%)$ FDs. In 15 patients $(29 \%)$, aneurysms were additionally coiled. The associations between procedural factors (number of FDs, additional coiling) and patient/aneurysm characteristics are summarized in On-line Table 1. More than 1 FD was used in patients presenting with a higher baseline mRS score $(P=.013)$, large aneurysm neck and size $(P<.001)$, and aneurysms located at the $\mathrm{BT}$ or $\mathrm{VB}(P<.001)$. Additional coiling was performed in patients presenting with a larger aneurysm neck and size $(P<.002)$, and the aneurysms were often located at the BT or $\operatorname{VB}(P=.001)$.

\section{Outcome Measures}

On-line Table 2 summarizes the correlations (Spearman $\rho$ ) between the outcome measures and the explanatory variables. For
Table 1: Patient data and characteristics of aneurysms treated with FD

\begin{tabular}{|c|c|}
\hline Characteristics & \\
\hline Patients (No.) & 52 \\
\hline Aneurysms (No.) & 52 \\
\hline Women (\%) & $21 / 52(41 \%)$ \\
\hline Mean age (range) (yr) & $54(16-79)$ \\
\hline \multicolumn{2}{|l|}{$\begin{array}{l}\text { Presentation/indications for } \\
\text { treatment (No.) (\%) }\end{array}$} \\
\hline Incidental finding/headaches & $20(38 \%)$ \\
\hline $\begin{array}{l}\text { Recurrent after coiling/coiling and } \\
\text { stenting/failed clipping }\end{array}$ & $16(31 \%)$ \\
\hline Cranial nerve deficit/mass effect & $14(27 \%)$ \\
\hline Stroke/transient ischemic attack & $7(13 \%)$ \\
\hline Acute SAH & $7(13 \%)$ \\
\hline \multicolumn{2}{|l|}{ Baseline mRS (No.) } \\
\hline mRS 0 & $20(38 \%)$ \\
\hline mRS 1-2 & $18(35 \%)$ \\
\hline mRS 3-5 & $14(27 \%)$ \\
\hline \multicolumn{2}{|l|}{ Aneurysm size $(n=51)(\%)$} \\
\hline$<5 \mathrm{~mm}$ & $4(8 \%)$ \\
\hline $5-9.9 \mathrm{~mm}$ & $13(25 \%)$ \\
\hline $10-20 \mathrm{~mm}$ & $17(33 \%)$ \\
\hline$>20 \mathrm{~mm}$ & $17(33 \%)$ \\
\hline \multicolumn{2}{|l|}{ Aneurysm neck size (mm) } \\
\hline Mean (range) & $17.5 \pm 17.7(2-90)$ \\
\hline \multicolumn{2}{|l|}{ Location (No.) (\%) } \\
\hline V4 segment of vertebral artery & $20(38 \%)$ \\
\hline VB junction & $11(21 \%)$ \\
\hline BT & $15(29 \%)$ \\
\hline PCA & $6(12 \%)$ \\
\hline \multicolumn{2}{|l|}{ Morphology (No.) (\%) } \\
\hline Wide-neck saccular & $12(23 \%)$ \\
\hline Fusiform/dissecting & $40(77 \%)$ \\
\hline \multicolumn{2}{|l|}{ Treatment } \\
\hline No. of flow diverters & $1.4 \pm 0.7$ (range, $1-3)$ \\
\hline No. of additional coiling & $15(29 \%)$ \\
\hline
\end{tabular}

clarity, analyses of the outcome measures are listed in the temporal order of assessment.

\section{Procedural Complications}

Technical problems and procedural complications were encountered in $9(17 \%)$ of 52 patients ( $95 \%$ CI, 8.2\%-30.3\%). Technical problems were the following: breakage of the pusher wire of the Surpass FD in 1 and damage of Surpass FD during positioning in 1. Procedural complications were the following: dissections along the arterial access in $3(6 \%)$, procedural thrombus formation along the parent vessel in $2(4 \%)$, rupture of the target aneurysm during FD placement in $1(2 \%)$, and infarction of the medulla oblongata related to takedown of the contralateral vertebral artery in a large VB junction aneurysm in 1 (2\%) (case example 2, Fig 2). Five of these patients remained clinically stable during follow-up. One patient worsened clinically (mRS $1-3$, case example 1 ), and 3 patients died during follow-up. As seen in On-line Table 2, procedural complications were correlated with age. Procedural complications did not differ with aneurysm location $(P=.304$, Kruskal-Wallis analysis of variance [KWANOVA]). Univariate logistic regression did not identify any significant predictor $(P>.085)$. Age, baseline $\mathrm{mRS}$, and number of FDs were eligible for inclusion $(P<.2)$ in the multivariable model (On-line Table 3). The multivariable logistic regression model did not identify any significant predictor. 

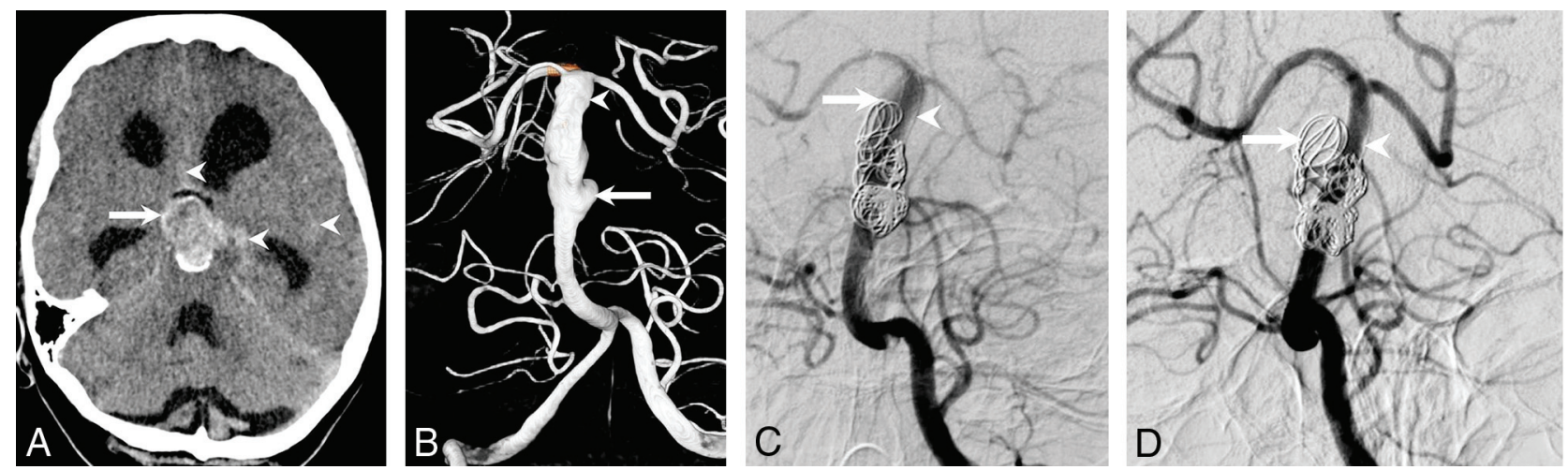

FIG 1. A 51-year-old woman presenting with Hunt and Hess grade 4 subarachnoid hemorrhage from a previously diagnosed fusiform aneurysm of the basilar trunk. A, Unenhanced CT scan shows diffuse SAH (arrowheads). The arrow indicates the partially thrombosed, fusiform aneurysm. B, The 3D reconstruction image shows a fusiform aneurysm (arrowhead) of the basilar artery with saccular components (arrow). C, Angiogram after placement of a Surpass FD (arrowhead) from the P1 segment of the right PCA to the midbasilar level. Additional coils were implanted. Note filling of the aneurysm (arrow). D, The 6-month follow-up angiogram shows complete occlusion of the aneurysm (arrow) and some intimal hyperplasia along the flow diverter (arrowhead). The mRS had changed from 2 before the SAH to 4 during follow-up. The clinical deterioration was most likely related to the $\mathrm{SAH}$. No new infarcts occurred in the posterior circulation after implantation of the flow diverter.
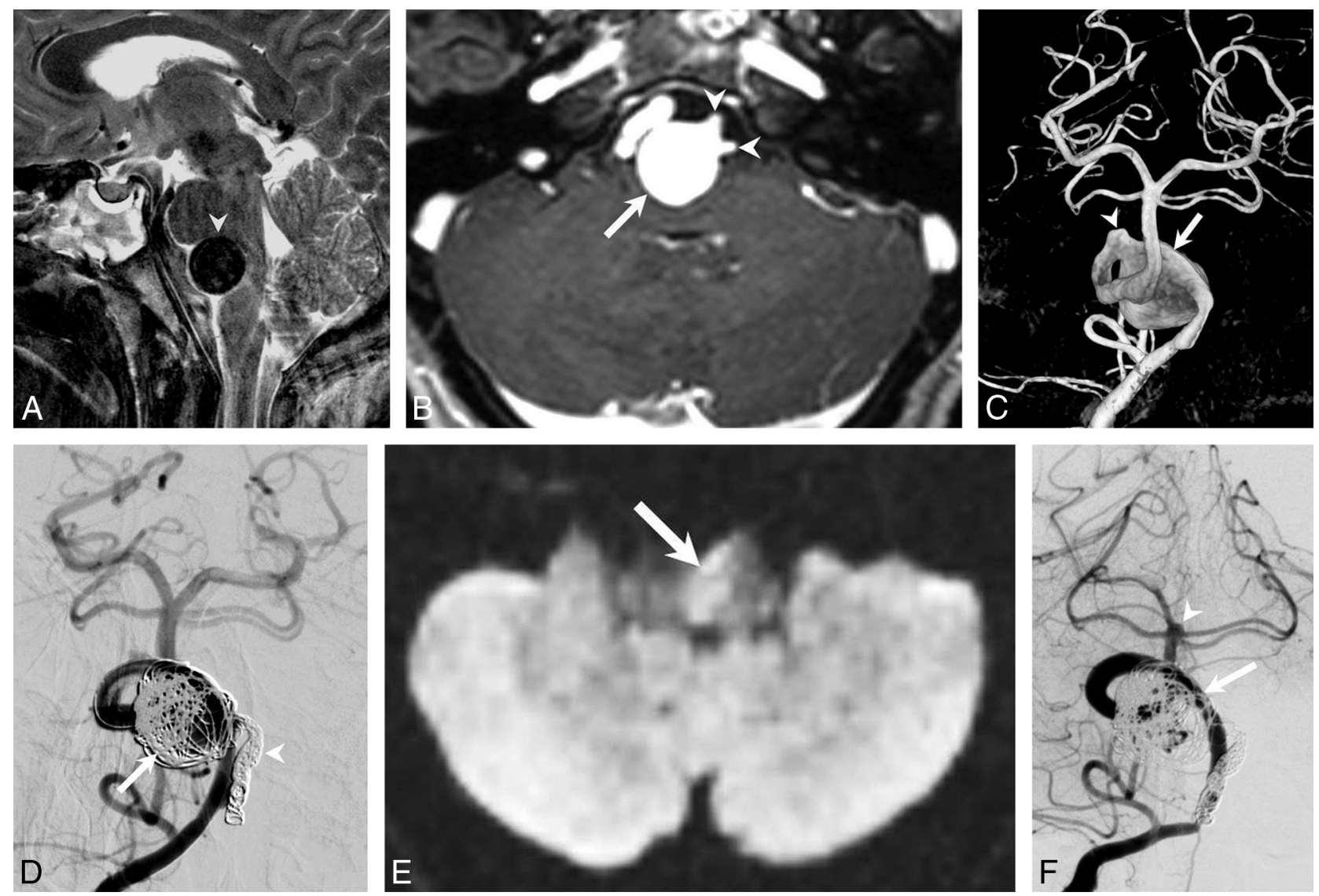

FIG 2. A 29-year-old man presenting with headaches and mild ataxia (mRS 1). A, Sagittal T2-weighted image shows a 32-mm vertebrobasilar junction aneurysm (arrowhead) compressing the medulla. B, Axial T1-weighted image with contrast shows the saccular aneurysm (arrow) and the 2 vertebral arteries (arrowheads) joining the aneurysm. C, The 3D reconstruction image shows a wide-neck (arrow) vertebrobasilar aneurysm with additional dysplastic segments (arrowhead) along the course of the basilar artery. D, Angiogram after placement of 2 Surpass FDs from the midbasilar level to the right vertebral artery. Additional coils were implanted (arrow). The distal segment of the left vertebral artery is occluded with coils (arrowhead). The patient was extubated 2 hours after the treatment and presented with paraplegia and respiratory insufficiency. E, Axial diffusion-weighted MR image $(b=1000)$ reveals a medullar infarct (arrow) within the vascular territory of the left anteromedial group of medullary arteries, most likely related to the voluntary occlusion of the left vertebral artery. $F$, The 6-month follow-up angiogram shows complete occlusion of the aneurysm (arrow). The left posterior cerebral artery is occluded (arrowhead). The time-of-flight MR angiography shows good collateral filling of the left posterior cerebral artery via the left posterior communicating artery (not shown). Clinically, the patient has improved. He now presents with a mild hemiparesis, slightly slurred speech, and ataxia (mRS 3). 


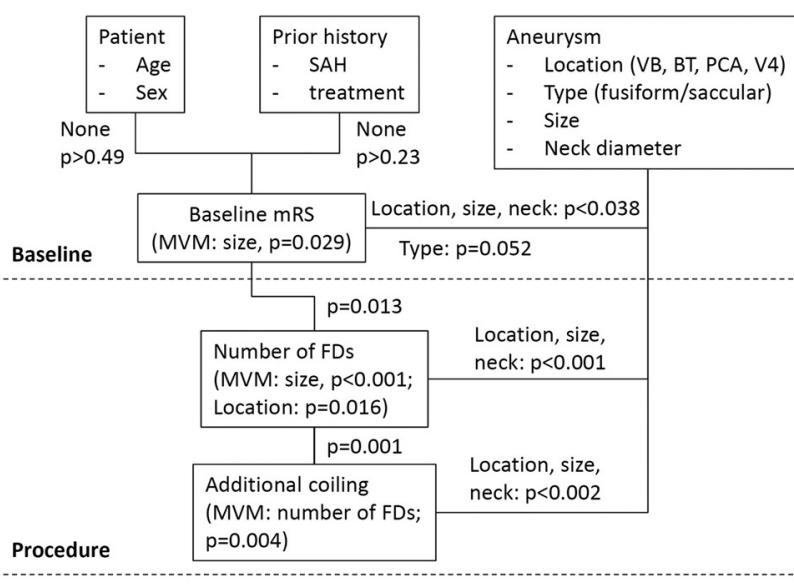

Outcomes (variables that were significant in univariate and multivariable models)

\begin{tabular}{|l|l|l|}
\hline Outcomes & Univariate models & Multivariable model \\
\hline Complications & None & None \\
\hline $\begin{array}{l}\text { New deficits at 24 } \\
\text { hours }\end{array}$ & $\begin{array}{l}\text { Aneurysm size, neck, location and } \\
\text { baseline mRS }\end{array}$ & Baseline mRS \\
\hline $\begin{array}{l}\text { mRS at hospital } \\
\text { discharge }\end{array}$ & $\begin{array}{l}\text { Aneurysm size, neck, location, } \\
\text { baseline mRS, and number of FDs }\end{array}$ & $\begin{array}{l}\text { Baseline mRS and } \\
\text { number of FDs }\end{array}$ \\
\hline $\begin{array}{l}\text { Complete occlusion } \\
\text { at follow-up }\end{array}$ & None & None \\
\hline $\begin{array}{l}\text { mRS at clinical } \\
\text { follow-up }\end{array}$ & $\begin{array}{l}\text { Aneurysm size, neck, location, } \\
\text { baseline mRS, and number of FDs }\end{array}$ & Baseline mRS \\
\hline Mortality & $\begin{array}{l}\text { Age, aneurysm size, neck, location, } \\
\text { number of FDs, dichotomized } \\
\text { baseline mRS (0-2 vs. 3-5) }\end{array}$ & $\begin{array}{l}\text { Dichotomized } \\
\text { baseline mRS, neck } \\
\text { and age (marginal) }\end{array}$ \\
\hline
\end{tabular}

FIG 3. Summary of statistical analysis. Univariate model results are along the connecting lines; multivariate model (MVM) results are within the box.

\section{Twenty-Four Hours Posttreatment}

Fourteen (27\%) of 52 patients (95\% CI, 15.6\%-41\%) experienced new neurologic symptoms, including the aforementioned patient with intraprocedural aneurysm rupture who died. For the remaining 13 patients, new neurologic symptoms ranged from minor cranial nerve deficits to tetraparesis. In 4 patients, new neurologic symptoms were related to procedural complications. Two patients with new neurologic symptoms 24 hours posttreatment had worsened mRS scores at follow-up (1-3 and 2-4), and 7 patients from this group died during follow-up. The $\mathrm{mRS}$ for the remaining 5 patients was either stable or improved. No symptomatic intracranial hemorrhage was observed immediately posttreatment. New neurologic deficits were positively correlated (On-line Table 2) with baseline mRS, BT location, aneurysm size, neck diameter, and the number of FDs, and they were negatively correlated with V4 location. All of the aforementioned variables were significant $(P<.036)$ in univariate logistic regression $(\mathrm{On}-$ line Table 3). Multivariable logistic regression identified only baseline $\mathrm{mRS}$ as a significant predictor $(P=.002$, On-line Table $3)$. The model satisfied the Hosmer-Lemeshow goodness-of-fit test $(P=.801)$. A unit (1-point) increase in baseline mRS was associated with a higher likelihood for new neurologic deficits at 24 hours posttreatment (OR, 2.28; 95\% CI, 1.60-3.82).

\section{Hospitalization and Discharge}

During hospitalization, another patient died following a medullary infarct. Four patients were discharged with deterioration of

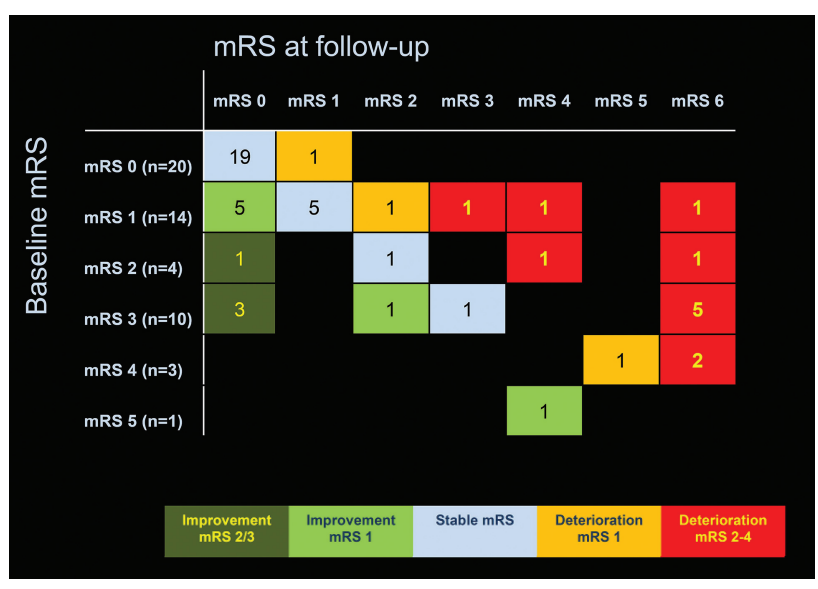

FIG 4. Shift from baseline mRS to $\mathrm{mRS}$ at follow-up $(n=52)$.

their clinical statuses, of which 3 were related to ischemic stroke. Another patient with a ruptured aneurysm and subarachnoid hemorrhage (Hunt and Hess grade 3) presented with clinical worsening associated with severe vasospasm. The remaining patients were either clinically stable $(39 / 50,78 \%)$ or had improved neurologically $(7 / 50,14 \%)$. The $\mathrm{mRS}$ score at discharge was positively correlated with baseline mRS, VB location, aneurysm size, neck diameter, and number of FDs, and it was negatively correlated with V4 location (On-line Table 2). Because the proportional odds assumption $(P<.001)$ could not be satisfied, generalized linear models were used for analysis (On-line Table 4). The final model included baseline mRS $(P<.001)$ and number of FDs (2 versus 1 , adjusted $P=.008$ ) as significant predictors. The model satisfied the goodness-of-fit criterion $(P=.551)$. After we adjusted for the number of FDs, a unit (1-point) increase in baseline $\mathrm{mRS}$ was associated with higher values of $\mathrm{mRS}$ at discharge (OR, 7.59; 95\% CI, 3.6-15.98).

\section{Complete Occlusion}

Angiographic follow-up was available for 44 (92\%) of 48 surviving patients; the median (quartiles 1-3, [Q1-Q3]) follow-up duration was 11.3 months (5.9-12.7 months). Complete occlusion was observed in 29 (66\%; 95\% CI, 50.1\%-79.5\%) of 44 patients and was positively correlated with prior SAH (On-line Table 2). Complete aneurysm occlusion differed with neither aneurysm location $(P=.768$, KWANOVA) nor baseline mRS $(P=.075$, KWANOVA). Neither univariate logistic regression $(P>.144)$ nor Kaplan-Meier analysis $(P>.194)$ identified any significant predictor.

\section{mRS at Clinical Follow-Up}

The median (Q1-Q3) follow-up duration was 11.3 months (5.912.7 months). Figure 4 shows the shift in mRS from baseline to follow-up. The mRS at follow-up was positively correlated with baseline mRS, VB location, aneurysm size, neck diameter, and number of FDs, and it was negatively correlated with V4 location and follow-up duration (On-line Table 2). The mRS at follow-up $(P<.009$, KWANOVA $)$ and the difference in mRS between follow-up and baseline $(P=.008$, KWANOVA) varied with aneurysm location. Because the proportional odds assumption $(P=$ .001 ) could not be satisfied, generalized linear models were used 
Table 2: Patients with fatal outcomes ${ }^{a}$

\begin{tabular}{|c|c|c|c|c|c|c|c|c|}
\hline Sex & $\begin{array}{l}\text { Age } \\
\text { (yr) }\end{array}$ & $\begin{array}{l}\text { Baseline } \\
\text { mRS }\end{array}$ & Location & Type & $\begin{array}{c}\text { Size } \\
(\mathrm{mm})\end{array}$ & $\begin{array}{l}\text { No. of } \\
\text { FDs }\end{array}$ & Cause of Death & $\begin{array}{c}\text { Survival } \\
\text { Time (days) }\end{array}$ \\
\hline M & 60 & 3 & BT & Fusiform & 10 & 1 & Ischemic stroke $48 \mathrm{hr}$ after FD treatment & 2 \\
\hline M & 58 & 4 & BT & Fusiform & 55 & 1 & Rupture during placement of the FD & 7 \\
\hline M & 64 & 2 & VB junction & Fusiform & 90 & 1 & Medullary infarct & 15 \\
\hline M & 55 & 3 & BT & Fusiform & 39 & 3 & Stable mRS at discharge; acute brain stem infarct & 50 \\
\hline M & 57 & 3 & VB junction & Fusiform & 40 & 3 & $\begin{array}{l}\text { Stable mRS at discharge; acute gastrointestinal bleed; } \\
\text { clopidogrel was stopped; died of urosepsis }\end{array}$ & 170 \\
\hline M & 59 & 4 & VB junction & Saccular & 55 & 3 & $\begin{array}{l}\text { Improved mRS at discharge (mRS 3); follow-up DSA at } \\
7 \text { months; aneurysm patent ( }>50 \% \text { ); at } 8 \text { mo, sudden } \\
\text { clinical impairment }\end{array}$ & 242 \\
\hline M & 74 & 3 & BT & Fusiform & 28 & 2 & Stable mRS at discharge; died of pneumonia & 388 \\
\hline $\mathrm{F}$ & 75 & 3 & VB junction & Fusiform & 30 & 2 & $\begin{array}{l}\text { mRS } 5 \text { at discharge to a tertiary care facility; } \\
\text { died of pneumonia }\end{array}$ & Unknown \\
\hline $\mathrm{F}$ & 65 & 1 & Vertebral artery & Fusiform & 8 & 1 & $\begin{array}{l}\text { Stable } \mathrm{mRS} \text { at discharge; died from complications } \\
\text { related to brain tumor treatment }\end{array}$ & Unknown \\
\hline
\end{tabular}

${ }^{a}$ All patients had presented with unruptured aneurysms.

for analysis. The final model (On-line Table 4) included baseline mRS $(P<.001)$ and follow-up duration $(P=.005)$ as significant predictors and satisfied the goodness-of-fit criterion $(P=.182)$. After we adjusted for follow-up duration, a unit (1-point) increase in baseline mRS was associated with higher values of mRS at follow-up (OR, 2.93; 95\% CI, 1.79-4.79). If we restricted the analysis to surviving patients $(n=43)$, the baseline mRS was the only significant predictor of mRS at follow-up $(P=.002)$ and a unit (1-point) increase in baseline mRS was associated with higher values of mRS at follow-up (OR, 2.53; 95\% CI, 1.42-4.51). Of 7 patients with SAH, 2 had an improved mRS (1-0,3-0), 4 had a stable $\mathrm{mRS}$ ( 0 in 3,3 in 1$)$, and 1 had a deteriorated $\mathrm{mRS}(2-4)$.

\section{Mortality and Morbidity}

The overall morbidity and mortality rate in our series was 14 of 52 (27\%). Nine of 52 patients died, resulting in an all-cause mortality rate of $17 \%(95 \% \mathrm{CI}, 7 \%-27.6 \%)$. Their clinical course is summarized in Table 2; the baseline mRS scores were $1(n=1), 2(n=$ $1), 3(n=5)$, and $4(n=2)$. More important, asymptomatic patients had $5 \%$ morbidity and $0 \%$ mortality, while symptomatic patients had morbidity and mortality rates of $44 \%$ and $28 \%$, respectively.

Mortality was positively correlated with baseline mRS, age, VB location, aneurysm size, neck diameter, and number of FDs and was negatively correlated with follow-up duration (On-line Table 2). The baseline mRS was dichotomized at 2 (inclusive) because there were no deaths in patients with baseline mRS scores of 0 and 5. Kaplan-Meier univariate analysis indicated age $(P=.018)$, aneurysm size $(P=.01)$, number of FDs $(P=.001)$, dichotomized baseline mRS $(P=.001)$, and aneurysm location $(P=.02)$ as significant, with neck diameter being marginal $(P=.06)$. All of the aforementioned variables were included in the Cox proportional hazards model with stepwise selection, which identified dichotomized baseline mRS $(P=.003)$ and neck diameter $(P=$ $.004)$ as significant, with age being marginal $(P=.072)$. Supremum tests for the functional form $(P>.358)$ and the proportional hazards assumption $(P=.444)$ were satisfied. After we adjusted for age and neck diameter, a baseline mRS of 3-5 was significantly $(P=.003)$ associated with a higher hazard ratio (HR) for death, compared with a baseline mRS of $0-2$ (HR, 17.11; 95\% CI, 2.69-109.02). During a median (Q1-Q3) follow-up of 11.6 months (5.9-13.3 months), 2 (5.3\%) of 38 patients with baseline mRS scores of $0-2$ and 7 (50\%) of 14 patients with baseline mRS of 3-5 died. Among the surviving patients, the median (Q1-Q3) follow-up durations were 11.7 months (8.5-12 months) and 11.9 months (8.6-14.6 months) for baseline mRS scores of 3-5 and $0-2$, respectively. Longer follow-up of patients in this cohort is needed to estimate survival times. Among the 43 patients surviving at the last follow-up, 6 had $\mathrm{mRS} \geq 3$, resulting in a morbidity rate of $14 \%$ (95\% CI, 3.6\%-24.3\%). Of the 6 surviving subjects with mRS 3-5 at follow-up, 3 worsened from the baseline mRS $(1 \rightarrow 3,1 \rightarrow 4$, and $2 \rightarrow 4), 2$ were stable $(3 \rightarrow 3$ and $4 \rightarrow 4)$, and 1 subject showed improvement $(5 \rightarrow 3)$. None of the subjects with a baseline mRS of zero had mRS 3-5 at follow-up. Of the 38 subjects with a baseline $\mathrm{mRS}$ of $<3$ and excluding the 2 mortality events, the morbidity rate was $8.3 \%(3 / 36)$. Of the 14 subjects with baseline mRS scores of 3-5, there were 7 deaths and 3/7 (43\%) surviving subjects had mRS scores of 3-5 at follow-up. Because only 6 patients had $\mathrm{mRS} \geq 3$ at follow-up, statistical analysis was not pursued.

\section{DISCUSSION}

To the best of our knowledge, this is the largest study to date to report exclusively on FD treatment of posterior circulation aneurysms.

The overall morbidity and mortality rate in our series was $27 \%$. Most important, asymptomatic patients had 5\% morbidity and $0 \%$ mortality, while symptomatic patients had morbidity and mortality rates of $44 \%$ and $28 \%$, respectively. A recent meta-analysis on flow-diverter treatment of posterior circulation aneurysms, including 220 patients with 225 posterior circulation aneurysms, reported morbidity and mortality rates of $6 \%$ and $15 \%$, respectively. ${ }^{5}$ The morbidity and mortality rates reported in the underlying studies ranged from $0 \%$ to $71 \%$ and $0 \%$ to $57 \%$, respectively. The variability in outcomes clearly reflects the heterogeneity of the underlying pathology.

Most posterior circulation aneurysms treated in our series were fusiform or saccular sidewall aneurysms and most likely dissecting aneurysms. Mizutani et $\mathrm{al}^{9}$ proposed a comprehensive classification of dissecting aneurysms. Type 1 corresponds to classic acute dissecting aneurysms, the pathogenesis of which is char- 
acterized by acute widespread disruption of the internal elastic lamina without intimal thickening. These aneurysms often have an ominous course with SAH. Type 2 aneurysms are segmental ectasias that have an extended or fragmented internal elastic lamina with intimal thickening. The luminal surface of the thickened intima is smooth without thrombus formation, and these aneurysms most often are located within the distal vertebral artery. Type 3 aneurysms are dolichoectatic dissecting aneurysms, pathologically characterized by fragmentation of the internal elastic lamina, multiple dissections of thickened intima, and organized thrombus in the lumen. Most of these lesions are symptomatic and progressively enlarge with time. ${ }^{9}$

For treatment of acutely ruptured type 1 aneurysms of the posterior circulation, stent-assisted coiling has become the treatment of choice. ${ }^{10}$ There is sparse literature on the use of FDs in this clinical setting. In our study, FDs alone and FDs in combination with coils resulted in acceptable clinical outcomes.

Patients with type 2 aneurysms are often asymptomatic at presentation. In our series, we observed no stroke-related symptoms related to FD treatment in this subset of patients, though a large number of perforating arteries were covered by the Surpass FD-a device with the highest mesh density available on the market. ${ }^{6}$ The clinical outcome was comparable with that of FD treatment of aneurysms in the anterior circulation. ${ }^{1}$ The notion that an increased risk of perforator occlusion in the posterior circulation may warrant caution in the use of FDs is not supported in this particular indication. In those cases in which perforators are directly covered by FD stents, those perforators most likely remain open. FDs seem to be a reasonably safe and effective treatment for type 2 aneurysms. There is some evidence that acute intervention is not always required, and close follow-up with antithrombotic therapy is a reasonable option. ${ }^{1-13}$ Yet, we lack greater knowledge on the clinical course of these potentially benign vascular lesions; this issue complicates any meaningful risk-benefit analysis of FD treatment in this clinical setting.

The high morbidity and mortality rates in our series were related to the treatment of patients with symptomatic type 3 aneurysms. These aneurysms are typically located at the level of the VB junction or the basilar trunk. In these large fusiform aneurysms that often present with a mural hematoma, perforators are displaced laterally and are at a distance from the FD device; this scenario results in eventual perforator occlusion. In addition, multiple overlapping devices may have a detrimental effect on incidentally covered small perforators. ${ }^{2}$ The results of our study and the review of the scarce literature illustrate the challenge of FD treatment of posterior circulation aneurysms. Type 3 aneurysms in the posterior circulation commonly are symptomatic due to ischemic strokes or may be diagnosed due to cranial nerve palsy, brain stem compression, obstructive hydrocephalus, SAH, or hemorrhages into the vessel wall. If left untreated, the natural history of these aneurysms is poor, with mortality rates between $23 \%$ and $35 \%$ in 5 years. ${ }^{14,15}$ Mortality increases significantly to $80 \%$ for untreated giant aneurysms in the posterior circulation. ${ }^{16}$ FD treatment in this setting, however, has a high risk of permanent morbidity and mortality, as our study demonstrates. It remains debatable whether intervention with an FD offers an improvement over the natural history of these lesions. ${ }^{17}$ In a small subset of patients with asymptomatic type 3 aneurysms (fusiform, located at the VB junction or basilar trunk, \pm the presence of an intramural hematoma) clinical results were surprisingly good.

It has been common practice to refrain from treating patients with large, fusiform, or partially thrombosed aneurysms in the posterior circulation as long as they are asymptomatic. Patients are often told to return for treatment only when they become symptomatic. Our analysis indicates that it may be safer to offer these particular patients early treatment. When treating these lesions, FD systems with longer single implants may be more advantageous than using multiple telescoping devices. In univariate analyses, our study showed that an increasing number of FDs was associated with poorer outcomes, including new neurologic deficits at 24 hours, higher mRS at follow-up, and mortality, but not in multivariable analyses. Patients treated with an increasing number of FDs presented with a higher baseline mRS score and larger aneurysm dimensions (On-line Table 1), and these baseline patient and aneurysm characteristics were contributors to poorer outcomes in multivariable analyses. Also, additional coiling was performed in patients presenting with larger aneurysm dimensions, and they were treated with $>1$ FD (On-line Table 1). These factors contribute to the observed results in multivariable analyses. Finally, the importance of adequate and long-term dual antiplatelet therapy has already been discussed in previous publications and cannot be emphasized enough. ${ }^{2,17}$

The angiographic complete occlusion rate in our series of $66 \%$ (29/44 cases; 95\% CI, 50.1\%-79.5\%) had overlapping 95\% confidence intervals with the meta-analysis of Wang et $\mathrm{al}^{5}$ (84\%; 95\% CI, 68\%-94\%). Any differences might be explained by the higher rates of giant and fusiform aneurysms in our patient cohort, with $33 \%$ versus $23 \%$ and $77 \%$ versus $66 \%$, respectively. ${ }^{5}$

Our study has various inherent limitations. It was designed as an international multicenter study in which patient selection was heterogeneous. Monitoring the dual antiplatelet therapy response was not standard procedure in all of the participating centers. Clinical and angiographic end points were self-reported by the institutions. In mRS at follow-up, we might have missed ischemic events related to FD treatment that did not lead to a deterioration of $\mathrm{mRS}$ score.

\section{CONCLUSIONS}

The mortality rate was comparable with that in prior studies, and angiographic results showed a high occlusion rate for this subset of complex aneurysms. Clinical results of FD treatment depend very much on patient selection, with poorer outcomes related to the treatment of aneurysms in patients with higher baseline mRS scores.

\section{ACKNOWLEDGMENTS}

The authors thank Mrs Dagmar Schnau for her invaluable assistance in editing and reviewing this manuscript.

Disclosures: Christian A. Taschner-RELATED: Consulting Fee or Honorarium: Stryker; UNRELATED: Consultancy: Acandis, Neuravi; Grants/Grants Pending: MicroVention, Acandis. * Joost de Vries—UNRELATED: Consultancy: Stryker Neurovascular; Grants/Grants Pending: Stryker Neurovascular; Payment for Development of Educational Presentations: Stryker Neurovascular; Travel/Accommodations/ Meeting Expenses Unrelated to Activities Listed: Stryker Neurovascular. Nobuyuki Sakai-UNRELATED: Grants/Grants Pending: Terumo, Comments: modest*; Pay- 
ment for Lectures including Service on Speakers Bureaus: Achieve, BioMedical Solutions, Codman Neuro, Medtronic, MicroVention/Terumo, Penumbra, Stryker Neurovascular, Comments: modest. Pedro Lylyk—UNRELATED: Travel/Accommodations/ Meeting Expenses Unrelated to Activities Listed: Surpass, Covidien, phenox, Cardiatis. Istvan Szikora-UNRELATED: Consultancy: Stryker Neurovascular, Codman Neuro, Medtronic, Sequent Medical. Stephan Meckel—UNRELATED: Board Membership: Acandis, Comments: consultancy and honoraria as member of the Scientific Advisory Board; Travel/Accommodations/Meeting Expenses Unrelated to Activities Listed: Covidien/Medtronic, MicroVention, Stryker Neurovascular, Acandis. Peter Kan-UNRELATED: Consultancy: consultant for Stryker Neurovascular. Matthew J. Gounis-RELATED: Grant: Stryker Neurovascular*; Consulting Fee or Honorarium: Stryker Neurovascular; UNRELATED: Consultancy: Codman Neuro; Expert Testimony: Harris Beach PLLC; Grants/Grants Pending: National Institutes of Health, Asahi, Blockade Medical, CereVasc LLC, Codman Neuro, Cook Medical, Gentuity LLC, Fraunhofer Society, InNeuroCo Inc, Lazarus Effect, Medtronic Neurovascular, MicroVention/Terumo, Neuravi, Philips Healthcare, Stryker Neurovascular, Wyss Institute*; Stock/Stock Options: InNeuroCo. *Money paid to the institution.

\section{REFERENCES}

1. Brinjikji W, Murad MH, Lanzino G, et al. Endovascular treatment of intracranial aneurysms with flow diverters: a meta-analysis. Stroke 2013;44:442-47 CrossRef Medline

2. Siddiqui AH, Abla AA, Kan P, et al. Panacea or problem: flow diverters in the treatment of symptomatic large or giant fusiform vertebrobasilar aneurysms. J Neurosurg 2012;116:1258-66 CrossRef Medline

3. Meckel S, McAuliffe W, Fiorella D, et al. Endovascular treatment of complex aneurysms at the vertebrobasilar junction with flow-diverting stents: initial experience. Neurosurgery 2013;73:386-94 CrossRef Medline

4. Ertl L, Holtmannspötter M, Patzig M, et al. Use of flow-diverting devices in fusiform vertebrobasilar giant aneurysms: a report on periprocedural course and long-term follow-up. AJNR Am J Neuroradiol 2014;35:1346-52 CrossRef Medline

5. Wang CB, Shi WW, Zhang GX, et al. Flow diverter treatment of posterior circulation aneurysms: a meta-analysis. Neuroradiology 2016;58:391-400 CrossRef Medline

6. Lieber BB, Sadasivan C. Endoluminal scaffolds for vascular recon- struction and exclusion of aneurysms from the cerebral circulation. Stroke 2010;41(10 suppl):S21-25 CrossRef Medline

7. Wakhloo AK, Lylyk P, de Vries J, et al; Surpass Study Group. Surpass flow diverter in the treatment of intracranial aneurysms: a prospective multicenter study. AJNR Am J Neuroradiol 2015;36:98-107 CrossRef Medline

8. Bonita R, Beaglehole R. Recovery of motor function after stroke. Stroke 1988;19:1497-500 CrossRef Medline

9. Mizutani T, Miki Y, Kojima H, Suzuki H. Proposed classification of nonatherosclerotic cerebral fusiform and dissecting aneurysms. Neurosurgery 1999;45:253-59; discussion 259-60 CrossRef Medline

10. Nam KH, Ko JK, Cha SH, et al. Endovascular treatment of acute intracranial vertebral artery dissection: long-term follow-up results of internal trapping and reconstructive treatment using coils and stents. J Neurointerv Surg 2015;7:829-34 CrossRef Medline

11. Kai $Y$, Nishi $T$, Watanabe $M$, et al. Strategy for treating unruptured vertebral artery dissecting aneurysms. Neurosurgery 2011;69:108591; discussion 1091-92 CrossRef

12. Shapiro M, Becske T, Riina HA, et al. Non-saccular vertebrobasilar aneurysms and dolichoectasia: a systematic literature review. J Neurointerv Surg 2014;6:389-93 CrossRef Medline

13. Kobayashi N, Murayama Y, Yuki I, et al. Natural course of dissecting vertebrobasilar artery aneurysms without stroke. AJNR Am J Neuroradiol 2014;35:1371-75 CrossRef Medline

14. Echiverri HC, Rubino FA, Gupta SR, et al. Fusiform aneurysm of the vertebrobasilar arterial system. Stroke 1989;20:1741-47 CrossRef Medline

15. Flemming KD, Wiebers DO, Brown RD Jr, et al. The natural history of radiographically defined vertebrobasilar nonsaccular intracranial aneurysms. Cerebrovasc Dis 2005;20:270-79 CrossRef Medline

16. Steinberg GK, Drake CG, Peerless SJ. Deliberate basilar or vertebral artery occlusion in the treatment of intracranial aneurysms: immediate results and long-term outcome in 201 patients. $J$ Neurosurg 1993;79:161-73 CrossRef Medline

17. Toth G, Bain M, Hussain MS, et al. Posterior circulation flow diversion: a single-center experience and literature review. J Neurointerv Surg 2015;7:574-83 CrossRef Medline 